ENCYCLOPEDDIE Encyclopédie berbère

BERBERE

$35 \mid 2013$

35| Oasitae - Ortaïas

\title{
Oranie / Oran (Linguistique)
}

Kamal Naït-Zerrad

\section{OpenEdition}

Journals

Édition électronique

URL : https://journals.openedition.org/encyclopedieberbere/2823

DOI : 10.4000/encyclopedieberbere.2823

ISSN : 2262-7197

\section{Éditeur}

Peeters Publishers

\section{Édition imprimée}

Date de publication : 1 juin 2013

Pagination : 5802-5805

ISBN : 978-2-7584-0184-1

ISSN : 1015-7344

\section{Référence électronique}

Kamal Naït-Zerrad, « Oranie / Oran (Linguistique)», Encyclopédie berbère [En ligne], 35 | 2013,

document 023, mis en ligne le 12 mars 2021, consulté le 17 février 2022. URL : http://

journals.openedition.org/encyclopedieberbere/2823; DOI : https://doi.org/10.4000/

encyclopedieberbere.2823

Ce document a été généré automatiquement le 17 février 2022.

(c) Tous droits réservés 


\title{
Oranie / Oran (Linguistique)
}

\author{
Kamal Naït-Zerrad
}

1 Dans son article sur la situation du berbère dans le département d'Oran, A. Basset fait le point en 1936 sur les connaissances de l'époque. On peut énumérer les zones ou îlots berbérophones en partant de l'est, à partir de Ténès :

- Les Achaacha, actuellement dans la daïra (sous-préfecture) de Achaacha, wilaya (préfecture) de Mostaganem : René Basset (1895) fournit des éléments sur cette langue qui semble être éteinte (Doutté \& Gautier 1913), donnée confirmée par A. Basset (1936).

- Les Beni Halima (daïra de Frenda, wilaya de Tiaret) : Doutté \& Gautier (1913) notent que ce parler se maintient encore mais qu'il est en voie de disparition. Il a été étudié par R. Basset (1895). En 1936, A. Basset signale qu'il est éteint.

- Le parler de Bettioua (Arzew, près d'Oran) : Doutté \& Gautier (1913) signalent qu'il semble éteint. Il s'agit d'un parler amené par un groupe de Rifains au milieu du XVIII ${ }^{\mathrm{e}}$ siècle et étudié par Biarnay (1910-1911).

- Les parlers à la frontière avec le Maroc :

Celui des Beni Mertgouch au Nord (fraction des Beni Iznassen* du Maroc);

Celui des Beni-Snous* et des Beni Bou Said dans la région de Tlemcen. Le parler des Beni Snous a bénéficié d'une très importance étude de Destaing $(1907,1911,1914)$ : grammaire, textes, dictionnaire. Les Beni Bou Saïd ont un parler très proche de celui des Beni-Snous.

Selon le recensement algérien de 1966 (RGPH), le seul qui ait comporté la rubrique "langue maternelle" depuis l'indépendance du pays, la wilaya d'Oran comptait : 28.510 berbérophones, sur un total de 955.838 habitants, soit un pourcentage $<3 \%$, ce qui paraît plausible si l'on ne prend pas en considération les nombreux Kabyles qui y sont installés, parfois depuis plusieurs générations (l'émigration des Kabyles vers l'ouest de l'Algérie - Tiaret, Oran...- est devenue significative après la première Guerre mondiale). Plus au sud, on trouve ce qu'on appelle traditionnellement les « ksours du Sud oranais " de part et d'autre de la frontière algéro-marocaine avec une vingtaine d'îlots en majorité du côté algérien (Bou Semghoun, Moghrar, Beni Ounif, Igli, etc.). Pour le 
Maroc, on citera en particulier l'oasis de Figuig* dont une description récente a été fournie par M. Kossmann (1997).

Tous les parlers de l'Oranie appartiennent au groupe dit zénète*. En dehors du sud oranais (Figuig), on manque donc cruellement d'enquêtes de terrain et de travaux récents publiés sur les parlers de cette région, maintenant profondément arabisée, qui constituait le coeur du monde zénète.

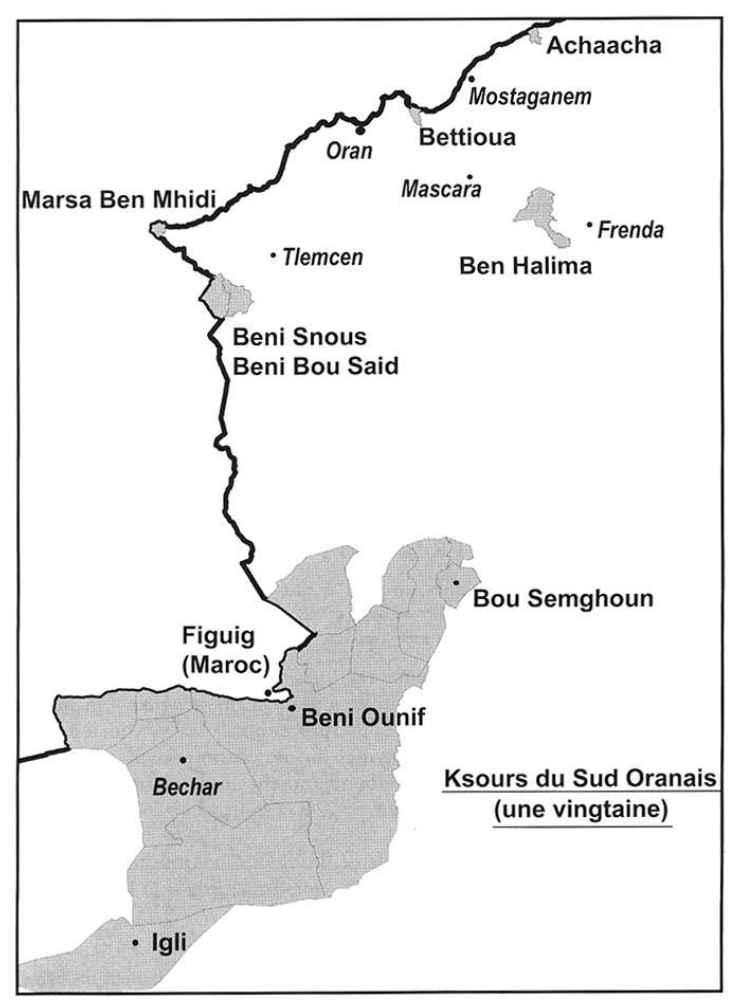

\section{Note sur l'étymologie du nom de la ville d'Oran}

7 La plus ancienne attestation du nom d'Oran semble se trouver dans Ibn Hawqal (2001, p. 74) dont l'ouvrage a été rédigé un peu avant l'an mil. Dans sa Description de l'Afrique septentrionale, El-Bekri (1913, p. 144), vers le milieu du XI ${ }^{\mathrm{e}}$ siècle, apporte des éléments complémentaires concernant en particulier la fondation de la ville. Dans les deux livres, la forme donnée est Wahrān. Plusieurs étymologies populaires ont été proposées pour ce toponyme. Mais le premier à avoir fait un rapprochement avec le nom du «lion » en berbère est Pellegrin (1949). On sait que le lion était présent en Oranie au moins depuis le néolithique. On peut en effet émettre l'hypothèse que Wahran est un composé dont le premier membre est soit le pronom/support de détermination pan-berbère wa « celui ; celui qui/que)», soit ewa " le lieu qui/que » (Foucauld III, 1457) qui est obligatoirement suivi d'un verbe. Le second élément (i/a)hran peut en conséquence être un nom ou une forme verbale participiale. On peut certainement écarter l'hypothèse verbale car aucun verbe ne semble correspondre au participe *ihran. L'hypothèse la plus probable reste donc la suivante : $w a[+n$ « de ; à »] + nominal

Un des noms du lion en berbère - certainement le plus ancien - est basé sur une racine HR/WR (cf. Chaker 2008) : 
ahar, pluriel : iharrən auquel on peut ajouter pour des parlers touaregs du Burkina

Faso et du Mali (Sudlow 2009, p. 88), le pluriel ehran (la voyelle initiale $e$ correspond

à un $i$ en berbère nord).

9 On postulera alors une séquence wa [n] әһrAП « celui des lions » (= (le lieu) des lions) qui devient par assimilation/amalgame wahran. On notera que cette construction avec un support pronominal wa + Nom est bien attestée en berbère pour des toponymes ou des anthroponymes (voir par exemple Foucauld 1940 où elle est abondamment illustrée ; sur ce modèle, voir aussi Chaker 1984, notamment p. 271 et Galand 1974, notamment §).

On signalera que l'on relève également Wahran comme toponyme ou oronyme ailleurs en Algérie et au Maroc, notamment dans le Rif.

\section{BIBLIOGRAPHIE}

BASSET A., « Situation actuelle des parlers berbères dans le département d'Oran », Revue Africaine, vol. 79-2, 1936, p. 1001-1006.

BASSET R., Etude sur la zenatia de l'Ouarsenis et du Maghreb Central, Paris, 1895.

BIARNAY S., « Etude sur les Bet't'ioua du Vieil-Arzew », Revue Africaine, vol. 54, 1910, p. 97-180, 301-342, 405-439/vol.55, 1911, p. 100-136,171-215, 327-347.

CHAKER S., Textes en linguistique berbère (Introduction au domaine berbère), Paris, Editions du CNRS, 1984 [chap. 14. "Onomastique berbère ancienne... ", reprend un article paru dans BCTH $\left(=2^{\circ}\right.$ Congrès d'Histoire et d'Archéologie de l'Afrique du Nord), n.s., 19, 1983 [1985], p. 483-497].

CHAKER S., «Lion : noms berbères du lion », Encyclopédie Berbère XXVIII-XXIX, Edisud, Aix-enProvence, 2008, p. 4420-4421.

DESTAING E., Etude sur le dialecte berbère des Beni-Snous, tome 1, Paris, Leroux, 1907.

DESTAING E., Etude sur le dialecte berbère des Beni-Snous, tome 2, Paris, Leroux, 1911.

DESTAING E., Dictionnaire français-berbère (dialecte des Beni Snous), Paris, Leroux, 1914.

DOUTTÉ E. \& GAUTIER E. F., Enquête sur la dispersion de la langue berbère en Algérie, Alger, 1913.

EL BEKRI, Description de l'Afrique septentrionale (trad. Mac Guckin De Slane), Paris, A. Maisonneuve, Paris, 1913.

FOUCAULD Ch. de : Dictionnaire touareg-français, I-IV, Paris, Imprimerie Nationale, 1951-1952.

FOUCAULD Ch. de : Dictionnaire abrégé touareg-français de noms propres (dialecte de l'Ăhaggar), Paris, Larose, 1940.

GALAND L., « Défini, Indéfini, Non-défini : les supports de détermination en touareg», BSLP, LXIX/1, 1974, p. 205-224.

IBN HAWQAL, Configuration de la terre : Kitâb Șûrat al-'ard. (Introduction et traduction avec index, J. H. Kramers et G. Wiet), Paris, Maisonneuve et Larose, 2001. 
KossmanN M. G., Grammaire du parler berbère de Figuig (Maroc oriental), Paris/Louvain, Peeters, 1997.

PELLEGRIN A., Essai sur les noms de lieux d'Algérie et de Tunisie : étymologie, signification, Tunis, SAPI, 1949.

RGPH = Recensement Général de la Population et de l'Habitat, 1966, série 1, vol. X (Wilaya d'Oran), RADP, Commissariat National au Recensement de la Population, Oran 1969.

SUDLow D., Dictionary of the tamasheq of North-East Burkina Faso, Rüdiger Köppe Verlag, Berber Studies, vol. 24, Köln, 2009. ${ }^{2}$

INDEX

Mots-clés : Algérie, Linguistique 